\title{
La communauté des Chanoines réguliers de l’Immaculée Conception au Manitoba
}

\section{Antoine Champagne}

Volume 37, 1970

La vie religieuse au Manitoba

URI : https://id.erudit.org/iderudit/1007285ar

DOI : https://doi.org/10.7202/1007285ar

Aller au sommaire du numéro

Éditeur(s)

Les Éditions Historia Ecclesiæ Catholicæ Canadensis Inc.

ISSN

0318-6172 (imprimé)

1927-7067 (numérique)

Découvrir la revue

Citer cet article

Champagne, A. (1970). La communauté des Chanoines réguliers de l'Immaculée Conception au Manitoba. Sessions d'étude - Société canadienne d'histoire de

l'Église catholique, 37, 229-246. https://doi.org/10.7202/1007285ar

Tous droits réservés @ Les Éditions Historia Ecclesiæ Catholicæ Canadensis Inc., 1972
Ce document est protégé par la loi sur le droit d'auteur. L'utilisation des services d'Érudit (y compris la reproduction) est assujettie à sa politique d'utilisation que vous pouvez consulter en ligne.

https://apropos.erudit.org/fr/usagers/politique-dutilisation/ 


\section{La communauté des Chanoines réguliers de l'Immaculée Conception au Manitoba}

On m'a demandé, à l'occasion du présent congrès, de présenter une étude sommaire sur la communauté des Chanoines réguliers de l'Immaculée Conception en général, d'exposer ce qu'elle peut avoir de particulier, de décrire en quelques mots quel fut son rôle au Canada et plus particulièrement au Manitoba.

Si j'ai accepté de préparer ces notes, malgré un état de santé qui me tient depuis longtemps éloigné de toute activité extérieure, c'est que j'ai cru rendre service à la cause de notre histoire qui, dans notre Manitoba plus qu'ailleurs, me semblait être aussi celle de l'Église et celle d'un sain patriotisme. N'est-ce pas là, au fond, le but principal de cette Société d'histoire et des congrès du genre de celui-ci ?

Je dirai d'abord quelques mots du fondateur Dom Gréa et de sa communauté en ce qu'elle a de particulier. Puis, je la montrerai à l'œuvre au Manitoba.

Si nous remontons à saint Augustin dont ils se réclament, au moins dans un sens large, nous voyons d'abord les Chanoines réguliers sous la forme d'un clergé vivant en commun autour de son évêque, sous une règle imposée par lui, s'occupant à la louange divine et accomplissant les œuvres du ministère paroissial communes à toutes les époques.

Plus tard, durant tout le Moyen Âge et après, on les trouve plus particulièrement dans les cathédrales et chargés de l'éclat des cérémonies liturgiques, ou dans des couvents disséminés dans divers pays et $\mathbf{y}$ accomplissant cette double fonction de la louange divine et du soin des âmes.

Plusieurs ajoutèrent à leur règle, selon les âges, un certain nombre de pratiques de la vie monastique.

Cet aperçu général nous amène à parler de Dom Gréa et de ses Chanoines réguliers.

Dom Gréa et les Chanoines réguliers de l'Immaculée Conception

Adrien Gréa, le futur fondateur des C.R.I.C., était né au Jura français en 1828, de Désiré-Adrien, qui fut un avocat réputé, député sous Louis-Philippe, puis membre de l'Assemblée nationale française en 1848, et de Françoise-Lucie Monnier. 
Adrien suivit d'abord les traces de son père, prenant sa licence en droit civil en 1845. Puis il se lança dans l'étude des anciens documents, obtenant un diplôme de l'Église des Chartes, à Paris, en 1850. La thèse qu'il présenta en cette occasion, et qu'il avait intitulée "Essai historique sur les archidiacres", l'avait mis en contact avec l'ancienne discipline de l'Église au Moyen Âge, et il s'était intéressé à cette époque particulière au point de presque s'y identifier dans la suite des années. On a dit de lui que c'était un homme du XIII ${ }^{e}$ siècle transplanté au $\mathrm{XIX}^{\mathrm{e}}$. Des malins ont même pu dire: "égaré au $\mathrm{XIX}^{\circ}$ "

Dès l'âge de 16 ou 17 ans, étant élève au collège de Besançon, où sa famille venait de se transporter pour mieux s'occuper de l'éducation de ses enfants, "il avait entendu les premiers appels du sacerdoce. La liturgie le passionnait: il aimait les messes chantées, il récitait une partie de l'office divin."

Le temps venu, il parla à son père de son désir d'être prêtre. Celui-ci, pris par la vie mondaine d'alors, l'accueillit assez mal. Adrien avait prévu cette opposition. Il acquiert une somme théologique de saint Thomas, petit format, et les volumes parus de la Patrologie de Migne, et il étudie la théologie dans sa chambre, guidé dans cette étude par un prêtre éminent, son directeur spirituel, l'abbé Eugène Hiron.

En 1856, sur le conseil de M. Caverot, le futur cardinal, il décide de passer outre à la résistance paternelle et part pour Rome, où il est le pensionnaire des bénédictins de Saint-Paul-hors-les-Murs, et prend son doctorat à la Sapience. Il est ordonné prêtre le 20 septembre suivant.

Bientôt, il est de retour dans son Jura et on le trouve aumônier aux Forges de Baudin, où un de ses ancêtres, Claude Jobez, avait établi en 1783 une fonderie de fer. Edmond Monnier, petit-fils de celui-ci et grand-père maternel d'Adrien, qui était propriétaire des Forges, avait décidé, comme Léon Harmel et vers le même temps, d'en faire une usine chrétienne où tous, patrons et ouvriers, formeraient comme une seule famille.

Une école cléricale y était née, avec le concours d'Adrien, en 1854, à l'occasion de la consécration d'une jolie église gothique, sous le vocable de l'Immaculée Conception, dont la définition allait avoir lieu le 8 décembre suivant. Dès lors, Adrien avait donné à ces fils d'ouvriers un règlement approuvé par l'évêque. Les enfants y chantaient tous les jours la messe et les vêpres et la liturgie y était en grand honneur. Sous sa direction immédiate, la tendance liturgique s'accentua. Le dimanche, tout le monde de l'usine chantait. Bientôt, les enfants revêtirent l'habit blanc, qu'ils gardèrent tout le jour. C'est vers ce temps, entre 1856 et 1863 , que vint à l'abbé Gréa l'idée de fonder une congrégation de chanoines réguliers, et on peut dire que ce fut là le berceau des Chanoines réguliers de l'Immaculée Conception. 
En 1863, $\mathrm{M}^{\mathrm{gr}}$ Nogret, évêque de Saint-Claude, offrit à l'abbé Gréa la charge de vicaire général, et, à ses objections, répondit: "C'est à Saint-Claude que vous établirez l'œuvre à laquelle vous vous destinez. »

Deux ans plus tard, la maîtrise de Baudin fut transférée à SaintClaude. Ici commence, à proprement parler, l'histoire des C.R.I.C.

En 1869, $M^{\text {gr }}$ Nogret part pour Rome, où il va assister au Concile du Vatican. L'abbé Gréa l'accompagne en qualité de théologien. Tous deux se montrent les défenseurs de l'infaillibilité pontificale. Remarquez, écrit $M^{\mathrm{gr}}$ Félix Vernet, l'historien de Dom Gréa, la singulière intuition de ce théologien de 42 ans, qui écrit en 1870: "Le XIX" siècle a été celui de l'Église universelle et de la papauté. Il fallait que la grandeur du Souverain Pontife fût d'abord mise en pleine lumière. Le $\mathrm{XX}^{\mathbf{e}}$ siècle sera celui de l'Église particulière: l'heure est venue de faire apparaître aux yeux du prêtre et des fidèles la vraie mission de l'épiscopat, en union avec le chef suprême de l'Église et dans la soumission à son autorité.»

En 1880, $\mathrm{M}^{\mathrm{gr}}$ Marpot succède à $\mathrm{M}^{\mathrm{gr}}$ Nogret malade et mal vu du gouvernement, qui avait inauguré sa politique antireligieuse. Dom Gréa est remplacé comme vicaire général.

Depuis 1865, il avait commencé à pratiquer avec quelques compagnons les observances de la vie canonique, matines à minuit, maigre et jeûnes traditionnels, etc. Le groupe avait fait un noviciat d'un an, suivi de vœux temporaires renouvelés annuellement pendant quelques années.

Le 8 septembre 1871, les cinq premiers Chanoines réguliers prononçaient leurs vœux perpétuels entre les mains de l'évêque. En 1873, ils prirent l'habit blanc pour ne plus le quitter. C'est le pape Pie IX qui voulut, quelques mois avant sa mort en 1878, que la communauté prît le titre officiel de Chanoines réguliers de "l'Immaculée Conception", et cela un peu contre l'idée de Dom Gréa, qui aurait préféré une appellation plus générale.

En 1879, la communauté comptait vingt-deux profès. En 1887, Léon XIII approuvait l'institut et remettait à plus tard l'approbation des constitutions, demandant que l'embryon actuel des règles fût rédigé d'une manière nouvelle et plus complète.

Tels furent les commencements des C.R.I.C., avec leur vie toute centrée sur la liturgie et la vie de pénitence des anciens ordres, avec " un ajouté bénédictin ", selon l'expression de Dom Gréa.

Tous les jours, la communauté chantait la messe et les vêpres à la cathédrale. Les matines étaient chantées la veille au soir, au même lieu les jours de fête. Le reste du temps, les exercices se faisaient à 
la chapelle de la communauté. La pénitence était celle des ordres monastiques: abstinence perpétuelle, jeûne du 14, septembre jusqu'à Pâques et deux fois par semaine, de la Pentecôte au 14 septembre.

La théologie se faisait dans la somme de saint Thomas, à laquelle s'ajoutaient certains traités plus modernes.

Dès 1878, les buts apparaissaient comme suit: 1. Liturgie. 2. Pénitence. 3. Soin des paroisses et vie des prêtres séculiers, avec la vie religieuse en plus.

En 1880, Dom Gréa accepte la première paroisse, Leschères, dans le diocèse de Saint-Claude.

1885 voit paraître l'ouvrage de Dom Gréa, De l'Église et de sa divine constitution, qui reçut des éloges de nombreux personnages ecclésiastiques éminents et qui a été réédité il y a quelques années.

En 1890, à la suite de restrictions dans l'office à la cathédrale, la communauté se transporte à Saint-Antoine en Dauphiné, dans le diocèse de Grenoble. Dom Benoît y arrive avec 24 novices en octobre. C'est là que la communauté va prendre définitivement son essor, en attendant d'être transportée en 1902 à Andora, en Italie, à cause de la persécution qui sévit en France contre les congrégations religieuses.

\section{Les Chanoines réguliers au Manitoba}

Mais, déjà, des événements qui nous touchent de plus près avaient commencé à se produire.

Dès 1887, devant les persécutions qui s'amorçaient, Dom Paul Benoît, jeune prêtre du Jura, entré dans la congrégation dix ans auparavant et devenu maître des novices, pensait au Canada comme refuge éventuel. Les années de Rome, où il avait pris un doctorat, l'avaient mis en contact avec des étudiants canadiens, et plusieurs avaient fait des séjours à Saint-Claude du Jura.

En 1890, avant l'installation à Saint-Antoine, il avait fait un voyage d'exploration au Canada, sur la demande de $\mathbf{M}^{\text {gr }}$ Taché, conseillé et aidé par le célèbre prêtre colonisateur, $\mathrm{M}^{\mathrm{gr}}$ Antoine Labelle, qui lui avait tracé un itinéraire et obtenu certains avantages pécuniaires. Il avait ainsi visité plusieurs évêques de la province de Québec, à Saint-Hyacinthe, Nicolet, Ottawa, etc., s'était rendu au lac Saint-Jean et dans l'Ouest jusqu'à Qu'Appelle et Fort Qu'Appelle, puis jusqu'à Saint-Léon et NotreDame-de-Lourdes, et avait fixé son choix, pour y établir les premiers Chanoines réguliers, sur ce coin pittoresque de la Montagne Pembina.

L'année suivante, il quittait Saint-Antoine le 16 avril avec trois compagnons et arrivait le 14 mai à Notre-Dame-de-Lourdes, petite mission née en 1883 et dépendant du curé de Saint-Léon, l'abbé Théobald 
Bitsche, qui s'occupait d'un immense territoire dans cette région du Manitoba.

Fonder une paroisse dans l'Ouest à cette époque, c'était non seulement être prêtre, mais aussi colonisateur. Les Chanoines réguliers seront l'un et l'autre dans toute la force du terme, mais je vous entretiendra uniquement ici du premier de ces deux rôles, c'est-à-dire de l'aspect religieux de cette fondation, avec ce qu'on y pressent déjà de nouveau et d'original, et laissant de côté à dessein l'aspect colonisation.

Les nouveaux arrivants étaient: Dom Paul Benoît, supérieur du groupe, le père Agnèce Patel, prêtre, le frère Placide Barthaut, sousdiacre, et le frère Félix Bugnon, convers.

Un brave colon, M. Charles Lafrenière, avait mis sa maison à la disposition du petit groupe, et était allé loger chez son fils Cyprien, à quelques milles de là.

On s'installa donc comme l'on put et aussitôt on commença, généreusement et gaiement, les exercices de la vie des C.R.I.C. La liturgie était au tout premier plan. Dès ce jour-là, la grand-messe et tout l'office du bréviaire furent chantés - rarement récités et seulement en cas de nécessité majeure - matines et laudes à minuit, les autres heures à divers moments de la journée; dès ce jour-là, les jeûnes et les abstinences furent observés, ainsi que les autres points de la règle.

Puis les Pères se mirent à organiser leur paroisse. Le service religieux se faisait dans leur maison, qui fut ainsi, en même temps qu'un monastère, la première église de Notre-Dame-de-Lourdes. Une grande croix en bois, avec inscription, en marque l'emplacement, à trois quarts de mille au sud du village, depuis 1942.

Il fallait maintenant construire: pour les Pères, un pauvre monastère, pour le bon Dieu, une pauvre église. On retrouva avec peine un long pieu fixé, l'année précédente au milieu de la forêt, par l'abbé Bitsche et Dom Benoît. Avec des secours apportés de France et d'autres reçus de $\mathrm{M}^{\mathrm{gr}}$ Taché ou de personnes généreuses, on construisit, en trois mois environ, une maison en planches, achetées à Somerset chez Allaire et Decosse, et une église en troncs équarris, qui mesurait un peu plus de de quarante par vingt pieds.

Le 15 août, $\mathrm{M}^{\mathrm{gr}}$ Taché avait érigé officiellement la paroisse. Date mémorable pour la petite colonie. Puis on avait dit la messe quelquefois dans la nouvelle église durant les jours qui suivirent.

Mais voici d'autres dates qui ne s'effaceront pas. Le 28 août, fête de saint Augustin, patron des Chanoines réguliers, on avait transporté au monastère les malles et le mobilier apportés de France et on s'était installé dans la nouvelle maison, heureux de se sentir chez soi. 
Ce bonheur fut de courte durée. Le lendemain, vers cinq heures de l'après-midi, alors que les Pères commençaient les vêpres, une femme de l'endroit, $M^{\mathrm{me}}$ François Chaperon, se précipite dans l'église tout effarée, en criant: "Le feu au monastère !" Déjà, les flammes léchaient les rebords du toit et, une heure plus tard, il ne restait plus, du monastère et de l'église, que des cendres fumantes. C'était le dénuement le plus complet. "Il ne nous reste plus, écrivait Dom Benoît, ni une paire de bas, ni une chemise de rechange. » Et ce qui rendait la chose plus tragique, c'est qu'on eut de fortes raisons de croire que le feu avait été allumé par une main criminelle. Telles sont les voies de Dieu: la croix prélude à la glorieuse résurrection.

Que faire devant ces ruines? Il ne restait que deux voies: se décourager et tout abandonner, ou bien recommencer. Avec le caractère que l'on connaît à Dom Benoît, on peut deviner ce qui arrivera. On recommencera.

Dom Benoît se mit à quêter dans les quelques paroisses qui existaient déjà aux environs: Saint-Boniface, Saint-Léon, Saint-JeanBaptiste, Saint-Pie-de-Letellier. Des dons arrivèrent de la province de Québec et de France, de ccux qui avaicnt déjà donné, les paurres, souvent, se dépouillant pour de plus pauvres. Mais sans attendre ces secours, on s'était mis à l'œuvre et des plans précis avaient été élaborés, pleins de sagesse et de prudence. Au lieu de deux constructions, on n'en aura qu'une, et au lieu de planches coûteuses, on se contentera des arbres de la forêt toute proche. C'est ainsi que, le 21 septembre, moins d'un mois après l'incendie, les premiers arbres étaient abattus; et, le 2 décembre, trois mois après l'incendie, on entrait dans la nouvelle maison. On l'appela "la maison-omnibus ", c'est-à-dire la maison à tous usages. Jamais appellation ne fut plus exacte. Le haut servait de résidence aux Pères. La partie inférieure était partagée par une cloison mobile qui permettait différents usages. Le dimanche, elle servait d'église. Les autres jours, la cloison se fermait et une moitié seulement était employée comme chapelle, tandis que l'autre servait à la fois de parloir, d'école, etc., en attendant de recevoir le bureau de poste, qui y fut installé plus tard. Bien des fois aussi elle servit d'hôtellerie, à l'arrivée de nouveaux colons. Cependant, la maison était loin d'être finie. On y travailla tout l'hiver, et durant le mois de janvier des ouvriers bénévoles vinrent s'offrir pour crépir l'intérieur avec de l'argile.

Une des principales préoccupations des Pères fut de dispenser un peu d'instruction aux enfants des colons. Dès le 8 décembre, Dom Benoît fit une offre dans ce sens aux paroissiens: "Nous n'avons pas d'école, leur dit-il, mais ne pourrait-on pas en créer une? Nous ne demandons aucun salaire, mais il nous faut un certain nombre d'enfants. La question de l'établissement d'une école cet hiver dépend de vous: 
voulez-vous envoyer dix ou douze enfants régulièrement?» Et le 22 décembre, le père Agnèce, remplacé parfois par Dom Benoît lui-même, commençait à faire la classe trois fois par semaine. Ce furent là les premiers maîtres d'école de Notre-Dame-de-Lourdes et non les moindres ni les moins méritants, et, au bout de six mois, les élèves qu'ils purent atteindre avaient fait des progrès faciles à constater et possédaient les premières notions des connaissances humaines et de la religion. Quelques années plus tard, les Chanoinesses des Cinq-Plaies venaient prendre la relève avec un dévouement égal et commençaient une œuvre qui n'est pas terminée et pour laquelle la paroisse ne saura jamais dire trop haut sa reconnaissance.

Cependant, on comprend que la maison-omnibus ne réglait pas le problème d'une église et qu'il fallait songer immédiatement à le résoudre. Le jour même où s'amorçait la question d'une église, Dom Benoît lançait l'idée d'une grande souscription en argent: "Il nous faut, disait-il, payer nos cendres... Il nous faut payer cette maison où nous sommes, et il faut penser à reconstruire. »

La souscription dans la paroisse ne donna pas, bien qu'il connût l'état de pauvreté des paroissiens, le résultat qu'il en attendait. Un montant de trois cents dollars en tout fut souscrit, dont une partie ne fut jamais versée.

Cela ne l'empêcha pas d'aller de l'avant avec une véritable audace. Le même jour, il lançait pour plus tard l'idée d'un couvent et même d'une école de frères. Comme on a vu, la première se réalisera bientôt, mais la deuxième n'eut pas de suite, malgré des démarches répétées faites par lui et par son successeur immédiat, le père Antoine Chalumeaux.

Il parlait aussi, ce jour-là, de l'achat d'un harmonium, qui viendrait rehausser les splendeurs du culte divin. Celui-ci, un gros harmonium Merklen de la meilleure qualité, arrivera bientôt et restera en service pendant plus de quarante ans, ne prenant sa retraite qu'en 1933.

Cependant, la vie de la petite communauté et la vie religieuse de la paroisse s'organisaient lentement et s'harmonisaient, comme des briques blanches et des briques de couleur s'harmonisent pour former un même mur.

Le tout était parsemé de joies et de tristesses.

Le 19 décembre, deux enfants étaient reçus dans la communauté et recevaient bientôt l'habit blanc des petits-frères. Bien qu'ils ne fussent pas de la paroisse et qu'ils n'aient fait qu'un stage assez court dans le monastère, Dom Benoît se plaisait à les considérer comme les prémices de l'école presbytérale par laquelle la paroisse devait alimenter plus tard la communauté nouvellement arrivée. D'autre part, ce n'était pas 
une pure perte, puisque l'un d'eux fonda une paroisse en Saskatchewan et en fut le curé pendant cinquante ans. Il est mort assez récemment à l'âge de 91 ans.

Parmi les tristesses, signalons le départ du père Placide Barthaut, qui partait le 30 octobre 1891, découragé et abattu, pour retourner en France. Puis, en juillet 1893, ce fut le tour du père Agnèce Patel qui, à la suite de plusieurs accidents, quittait Notre-Dame-de-Lourdes pour ne plus y revenir, malgré le désir qu'il en avait. Le brave père, qui desservait la paroisse de Saint-Léon depuis Notre-Dame-de-Lourdes après la mort de $M$. Bitsche, se gela un jour les deux pouces et dut se faire soigner à l'hôpital de Saint-Boniface. Plus tard, il avait reçu d'un jeune poulain un coup de pied en pleine poitrine et sa santé déclinait depuis longtemps. Plus tard, le frère Félix Bugnon quittait la communauté.

Ces tristesses ne furent pas, cependant, sans compensation, sinon toujours pour Dom Benoît et ses confrères, du moins aux yeux de Dieu. Le jour même du départ du frère Placide, arrivait de France le père Marie-Antoine Straub, qui sera pour Dom Benoît un compagnon et un ami fidèle et sa plus douce consolation dans l'épreuve. Ajoutons qu'en 1938 le père Patel était un curé fervent et respecté dans une paroisse de France, et le père Barthaut était devenu vicaire général à l'île Maurice, au sud-est du continent africain.

La vie des colons était dure. Les Pères, qui en avaient le souci spirituel, pratiquaient de cette vie toute la partie pénible et laborieuse quand les occupations du ministère, l'accomplissement de la règle, l'enseignement ou l'étude le leur permettaient.

Et il le fallait bien; car si c'était générosité, c'était aussi nécessité. Avec les ressources limitées des paroissiens, et donc de leurs pasteurs, ceux-ci n'auraient eu qu'à mourir de faim s'ils avaient dû se contenter de ce qu'on leur donnait. En 1891, la dîme, qui constituait alors le traitement du prêtre et qu'on payait en nature, se montait à deux cents minots de blé et quelques autres grains. Le blé se vendait alors cinquante sous le minot, cela faisait un peu plus de cent dollars pour quatre personnes, soit vint-cinq dollars pour chacune et pour l'année.

Quant aux revenus de l'église, ils étaient en proportion. Nous pouvons en juger par les quêtes de cette même année qui s'élevaient, pour les quatre derniers mois, à 33 cents ou sous par dimanche. En 1892, la moyenne est de 40 cents.

Il fallait donc trouver d'autres moyens de subsistance. Aussi Dom Benoît se préoccupa-t-il dès son arrivée de doter la communauté d'un patrimoine et recourut-il à l'aide charitable de $\mathrm{M}$. Bitsche qui jamais ne marchandait sa peine. Puisque le gouvernement se montrait généreux 
pour les colons et leur concédait des terres presque gratuitement, ils ne voyaient pas de raison pour que les Pères, s'ils remplissaient les obligations de tout le monde, n'eussent pas les mêmes droits. Cela n'allait pas, paraît-il, sans quelque difficulté. Mais bientôt, grâce aux bons offices de M. le sénateur Thomas-Alfred Bernier et de M. Alphonse Larivière, député de Provencher, plusieurs Pères purent avoir leur " homestead " ou concession gratuite de terrain, et c'est ce qui permit à la communauté de vivre et de se développer.

C'est ainsi que le Quart nord-est du 36-6-9 fut adjugé comme * homestead " régulier au père Agnèce Patel, qui se hâta d'en détacher quarante acres pour les donner à la paroisse et reçut sa patente ou titre de propriété en bonne et due forme. $\mathrm{M}^{\mathrm{gr}}$ Taché avait coutume, toutes les fois que la chose était possible, de réserver ainsi quarante acres pour les besoins des paroisses en formation.

Voilà pourquoi et comment les Chanoines réguliers, Dom Benoît payant d'exemple, défrichaient, labouraient, ensemençaient, bâtissaient. C'étaient vraiment les émules des anciens moines, qui défrichèrent l'Europe, en même temps qu'ils y faisaient pénétrer l'Évangile.

Ces toutes premières constructions, ces premiers efforts pour survivre, cette première organisation du service paroissial, d'une toute petite communauté avec sa règle moyenageuse faite d'austérités et de longues prières du jour et de la nuit, cet embryon d'école pour les petits, tout cela pourrait s'appeler «le commencement des commencements ».

Mais on ne pouvait en rester là et tout de suite il fallait songer à quelque chose de plus stable et plus permanent. Ce qui pressait le plus, avec les développements qui s'annonçaient, c'était une maison et un lieu de culte pouvant satisfaire aux besoins de l'avenir, au moins pour un certain temps. Dès le 8 décembre, fête de l'Immaculée Conception, patronne de la paroisse et de la communauté, Dom Benoît voulut lancer l'idée et commença son appel. Mais, bien qu'il ne fût pas facile à émouvoir, les épreuves par lesquelles il venait de passer eurent momentanément le dessus et, après les premières phrases, il se troubla et les sanglots l'étouffèrent. Il lui fut impossible de continuer. Pendant un long moment il attendit, puis retourna à l'autel où, d'une voix brisée, il réussit à entonner le credo. Il revint à la charge les dimanches suivants, puis convoqua une assemblée paroissiale où les paroissiens étudieraient les divers aspects de la question.

On élimina tout de suite l'idée de construire en planches, car les moyens ne le permettaient pas. La forêt avoisinantes fournirait la plus grande partie du matériel. Des troncs d'arbre équarris à la hache, de l'argile pour boucher les interstices, beaucoup de travail gratuit et 
donc de courage, telles seraient les principales ressources qui permettraient de mener à bonne fin l'œuvre entreprise. On bâtirait le monastère seulement, qui serait définitif, mais dont une partie servirait définitivement d'église.

Tout de suite, on se mit à l'œuvre. Deux ans et demi plus tard, le 28 août 1894, en la fête de saint Augustin, deuxième patron des C.R.I.C., le père Allard, o.m.i., administrateur du diocèse de SaintBoniface après la mort de $\mathrm{M}^{\mathrm{gr}}$ Taché, accompagné du R. P. Louis. prieur de la Trappe de Saint-Norbert, et de l'abbé Télesphore Campeau, curé de Saint-Alphonse, bénissait la nouvelle maison, en présence de la paroisse et de quelques prêtres du voisinage. Les Pères s'y étaient transportés l'année précédente et les exercices du dimanche s'y faisaient depuis quelques mois, pendant que continuaient les travaux.

C'était une grande maison en forme de croix grecque, pouvant, quand elle serait finie, loger une bonne quarantaine de religieux. La charpente et les murs consistaient en troncs de chêne équarris, le plancher et le toit étaient de planches, celui-ci étant recouvert de bardeaux. Le tout était surmonté d'une petite tourelle carrée que dominait la croix. Les Pères occupaient le bas, tandis que les offices religieux se faisaient dans la partie du haut, à laquelle on accéda bientôt par un double escalier extérieur.

La bâtisse servit à la communauté jusqu'en 1913, alors que l'on construisit le grand presbytère revêtu de briques, qui loge aujourd'hui l'administration du Foyer Notre-Dame, refuge de personnes âgées.

A l'automne de 1894, nous voyons donc la paroisse dotée des édifices nécessaires à son développement normal. Pendant qu'elle se développait ainsi matériellement, le progrès spirituel allait de pair. L'organisation se complétait tout doucement pendant que la vie religieuse évoluait au milieu d'un déploiement de chants et de cérémonies qu'on aurait vainement cherché ailleurs, même dans une cathédrale. La vie des C.R.I.C., telle que conçue par Dom Gréa et réalisée ici dès le commencement par Dom Benoît, qui surenchérissait encore sur le fondateur, comportait le chant intégral de l'office divin et de la messe ou des messes que réclamait la liturgie. Matines et laudes se chantaient à minuit, les autres heures et les messes s'espaçaient dans la journée. Je ne dirai pas que tout cela allait toujours selon toutes les règles de l'esthétique ou de l'art musical. Quand, dans les premières années, plusieurs Pères devaient s'absenter en même temps, ce qui arrivait souvent, et que l'unique Père restant chantait les matines ou la messe avec les deux petits postulants âgés de 10 ou 12 ans, ou encore avec le domestique M. Bœuf (quel nom pour un chantre!), dans des manuels polycopiés à la main et de mauvaise venue, je parierais volontiers que la mélodie devait s'éloigner quelque peu de celle de Solesme et que la 
langue de Cicéron devait souffrir quelques accrocs; mais il y avait là tant de piété et de bonne volonté que c'était bien, malgré tout, l' "opus divinum " et que le bon Dieu y trouvait sa gloire. On peut supposer cependant que, dans ce désert qu'était la paroisse de Lourdes, les paroissiens n'étaient pas tous des Pères du Désert; et les cérémonies paraissaient longues à quelques-uns. Aussi Dom Benoît ne manquait pas de gourmander ceux qui sortaient pendant l'office de sexte, ce complément nécessaire de la messe du dimanche, les "petites vêpres " comme il l'appelait, destinées à remplacer pour beaucoup les vêpres de l'après-midi.

La vie paroissiale évoluait dans cette liturgie comme le poisson dans l'eau. Celle-ci enveloppait, dominait, inspirait tout le reste et tout lui était subordonné. On voulait qu'il en fût de la vie de l'Église ici-bas conme de celle de l'Église du ciel, où la louange de Dieu est l'unique chose nécessaire.

Pour le reste, la vie paroissiale ressemblait à ce qui se fait partout ailleurs et la paroisse naissante s'organisait religieusement comme toute paroisse naissante. Baptêmes, mariages, sépultures, premières communions, confirmations, visites de paroisse, œuvres de la Propagation de la Foi et de la Sainte-Enfance, Association de la Sainte-Famille prirent naissance dans les deux premières années. La première retraite paroissiale eut lieu en 1893, prêchée par le père Joseph Cottet, qui avait amené d'Europe un groupe de colons. La deuxième eut lieu l'année suivante, prêchée par Dom Gréa, qui faisait sa première visite à ses fils du Canada et venait constater et bénir leur travail.

Là où on trouve plus d'originalité, c'est dans les faits divers qui surviennent et les traditions qui, dès les commencements, s'installent dans la paroisse.

La première, apportée peut-être de Lyon, où elle se fait depuis des siècles, est l'illumination des maisons en l'honneur de la Sainte Vierge, le jour de l'Immaculée Conception. Pour cette première fois, seule la tourelle du monastère fut ornée de banderolles et quelques bougies y brillèrent dans la nuit. Mais, depuis cette époque, toutes les maisons du village et plusieurs de la campagne s'embrasent chaque année de sept à huit heures du soir, au son de la cloche, pendant que dans l'église et sur la place, devant les statues de Marie, des chants latins et français retentissent en son honneur.

Autre tradition tout aussi délicieuse apportée de Rome par Dom Benoît et qui n'a pas été interrompue, pour autant que sache, pendant plus de cinquante ans, depuis le 25 décembre 1894. Dans l'après-midi de Noël, un petit prédicateur de dix ou douze ans, encadré des enfants de chœur, s'avance vers la Crèche, pendant que résonnent les couplets 
d'un vieux cantique, alterné par les petits garçons et les petites filles et annonçant le thème probable du sermon:

\author{
Allons tous à la Crèche \\ Entendre un beau sermon. \\ C'est le Sauveur qui prêche \\ Pour notre guérison. \\ Nous avons tous besoin \\ D'un médecin si sage, \\ Et le remède n'est pas loin \\ Pourvu que nous prenions le soin \\ D'en faire un bon usage.
}

Puis les chrétiens tièdes s'entendent dire de dures vérités: avares, ambitieux, jouisseurs, coquettes, tous reçoivent leur compte et sans ménagement. Le sermon qui suit a l'avantage de ne pas être long. De deux à quatre minutes suffisent à ce prédicateur d'occasion et la conclusion arrive, suivie de la bénédiction du Père Curé. Un ou deux autres cantiques pour finir: le tout a duré environ un quart d'heure. Suivent les vêpres de Noël et la bénédiction du Saint-Sacrement.

Le 24 juin 1893, on inaugura à Notre-Dame-de-Lourdes la fête du patron des Canadiens français par un usage importé de France et dont l'origine remonte au Moyen Âge: le feu de joie de la Saint-JeanBaptiste. Le bois ne manquait pas et, pendant de nombreuses années, c'était le bonheur des jeunes étudiants d'amasser un bûcher monstre et de regarder les flammes monter dans la nuit. Cet usage disparut plus tard faute de... combustible. Il fut remplacé par des démonstrations plus élaborées et qui sont tout à l'honneur de colons venus de France et de Suisse pour la plupart: messe solennelle et prédication de circonstance, défilé historique avec le petit saint Jean-Baptiste et ses quatre pages, joutes sportives et amusements divers, etc. Je crois que cette célébration se fait encore, avec des variantes.

Il y a lieu de mentionner, pour finir, le pèlerinage annuel que fit pendant de longues années la paroisse de Saint-Léon à Notre-Damede-Lourdes, vers le commencement de l'été, et qui date, si mes souvenirs sont exacts, d'avant 1900 et peut-être des années que nous venons de parcourir. Toute la paroisse de Saint-Léon s'ébranlait alors pour offrir ses hommages à la Vierge de Massabielle et lui demander ses faveurs. A un mille environ du village de Lourdes, le clergé local se rendait à la rencontre des pèlerins et une double procession s'organisait, avec croix et oriflammes, qui remplissait l'église pour la messe solennelle et la prédication de circonstance. Plusieurs faisaient la sainte communion, à cette heure tardive, ayant jeûné depuis la veille. On mangeait ensuite sous les arbres, chacun ayant apporté le nécessaire, et on flânait quelques heures, pendant que les plus fervents ou les plus éprouvés adressaient à la Vierge un supplément de prières. On repartait après la bénédiction du Saint-Sacrement, à une heure tardive de l'après-midi. 
A la longue, des groupes ou individus des paroisses voisines se joignirent aux pèlerins de Saint-Léon. On en vit de Saint-Claude, Haywood, Rathwell, Treherne, Somerset et Bruxelles. Quelques-uns vinrent à pied de vingt milles et plus, partant de chez eux aux toutes petites heures du matin. Aux chevaux et aux piétons succédèrent les premières autos. Mais quand celles-ci se furent multipliées, le pèlerinage perdit sa popularité et finit par disparaître. Cela se produisit vers les 1930 ou un peu avant.

On pourrait continuer, mais nous avons maintenant une bonne idée de cette paroisse d'Européens devenus Canadiens et de leur mentalité, de même que de celle de leurs pasteurs.

Il reste à mentionner la lutte scolaire pour la défense du français et de la religion à l'école, lutte qui dure au Manitoba depuis 1890 et dont les deux aspects se tiennent de très près dans notre province.

Les C.R.I.C. s'y donnèrent dès le commencement avec toute leur énergie et leur courage et toute la paroisse les suivit, sauf de très rares exceptions. Dom Benoît fut l'âme de cette lutte dans le territoire confié à ses soins, et ses successeurs C.R.I.C. ont marché sur ses traces, sous la direction de leurs chefs spirituels, $\mathrm{M}^{\mathrm{gr}}$ Langevin et $\mathrm{M}^{\mathrm{gr}}$ Béliveau, pour ne nommer que les plus anciens. La lutte se continue, comme l'on sait.

Pour ce qui est des premières années, il est impossible de ne pas mentionner ici deux autres lutteurs amis de Dom Benoît, soit M. Louis Hacault, qui s'était déjà battu vaillamment dans la libre Belgique, et M. Paul Tardivel, de Québec, ce Franco-Américain devenu Canadien, tous deux journalistes de qualité, et on croirait voir dans ces quatre vaillants soldats du Christ un généralissime, $\mathbf{M}^{\mathrm{r}}$ Langevin, assisté, de près ou de loin, par trois généraux d'armée, qu'il regardait comme ses aides les plus précieux et les plus dévoués.

Dois-je vous décrire maintenant une journée de Dom Benoît et de sa communauté, une des journées que je connais si bien, pour les avoir vécues pendant plus de huit ans?

A minuit exactement, le frère "excitateur " faisait retentir dans les chambres et les dortoirs le Benedicamus Domino, que deux religieux chantaient les jours de fête, et, après une toilette sommaire, on se rendait à l'église paroissiale, hiver comme été, pour le chant des matines et des laudes. Il gelait souvent à pierre fendre, car le feu venait seulement d'être allumé, mais bientôt le poêle ronflait et on se faisait rôtir copieusement par devant pendant qu'on gelait par derrière, cependant que les chants sacrés se déroulaient lentement. L'office durait une heure et trois quarts environ en moyenne, un peu plus aux jours de fête. On retournait ensuite au monastère où on faisait dans la salle commune une demi-heure de méditation. L'horloge marquait deux heures et un 
quart en moyenne. Tous alors regagnaient leur couchette consistant en une paillasse, oreiller également de paille, draps et couvertures en grosse laine, le coton étant trop moelleux et contraire à la mortification, le tout sur des planches que portaient des trétaux de bois ou de fer. Mais pendant que tous reposaient, Dom Benoît faisait encore une demi-heure de lecture d'Écriture Sainte, ce qui menait à deux heures trois quarts environ et parfois trois heures. Les premières heures de la journée étaient donc au bon Dieu.

A six heures moins un quart, nouveau réveil, toilette, chant de prime à l'église, avis, distribution du travail et souvent petite conférence par Dom Benoît, de dix à quinze minutes, à la salle du chapitre ou sacristie. Messes des prêtres, étude pour les autres, déjeuner consistant pour les profès en une tasse de café sucré, sans lait ni nourriture, les deux tiers de l'année environ. Aux jours de fête, "caritas ", c'est-à-dire café au lait avec une tranche de pain.

A sept heures trois quarts, chant de tierce, de la messe, et de sexte. Étude. Classes. Dom Benoît enseignait tous les jours la théologie et souvent d'autres matières, par exemple la philosophie ou les sciences naturelles.

A 11 heures, dîner ou collation. Lecture à haute voix, que les religieux faisaient à tour de rôle pendant quelques minutes, tout le temps du repas. Bénédicité et grâces chantées. Récréation aux repas les jours de grande fête ou à l'occasion du passage d'un visiteur de marque. On jeûnait tous les jours, sauf aux grandes fêtes, du 8 septem. bre jusqu'à Pâques. L'abstinence était perpétuelle pour tous les profès non dispensés. Dom Benoît ne goûta pas à la viande de 1877 à 1901 . Il fallut un ordre du médecin pour lui en faire prendre pendant la maladie grave qu'il fit alors. On peut en dire autant de plusieurs autres pères et frères.

Aux jours de grand jeûne, la collation consistait en un "mixte ", c'est-à-dire un bol de café, thé ou infusion, auquel on ajoutait une bonne quantité de chocolat et une tranche de pain. Aux autres jours de jeûne, la collation équivalait à un quart de repas.

Pendant le carême et aux jours où la liturgie comporte deux messes, les deux étaient chantées, la deuxième vers midi. Dom Benoît chantait cette messe tardive plus souvent qu'à son tour, se condamnant ainsi à un jeûne strict jusqu'à une heure tardive.

Après le repas ou la collation, première récréation de la journée, qu'il ne manquait jamais. Ensuite, il fait une heure de travail manuel. L'été, le plus souvent, il abattait les arbres dans la forêt toute proche, l'hiver, il dépeçait le bois pour les fournaises. Aux jours de congé, il prenait les mancherons de la charrue, suivait la herse ou faisait les foins, comme tout le monde. 
Après la récréation, étude ou travail de bureau et classes. La première partie de ce temps d'étude était consacrée à sa volumineuse correspondance, pour laquelle il employait volontiers l'aide d'un secrétaire, auquel il dictait lentement mais sans interruption. bureau.

A 4 heures, chant de none et des vêpres. Étude ou travail de

A 5 heures et demie, souper. C'était, pour les profès, l'unique repas de la journée et il le prenait copieux. Ensuite, récréation de $\mathbf{4 0}$ ou 45 minutes. Travail manuel ou étude.

A 7 heures et demie, chant de complies, précédé d'une conférence d'un quart d'heure à la salle du chapitre ou sacristie et d'un petit examen de conscience. Cette conférence était presque toujours faite par Dom Benoît.

A 8 heures, coucher, hiver comme été.

Entre temps, à toute heure de la journée, il recevait les paroissiens et réglait leurs affaires.

Les dimanches et jours de fête, il , avait prédication à la messe et aux vêpres, sans compter les réunions de confréries, dont une par dimanche. Dom Benoît faisait la moitié au moins des prédications. Elles étaient claires et pratiques.

Dans l'après-midi du dimanche, il y avait une grande conférence sur des sujets divers: vie religieuse, histoire locale, état religieux de la France. Dom Benoît en faisait le plus grand nombre. Certains Pères faisaient le reste, ou même parfois de jeunes religieux. Dans ce cas, surtout lorsqu'ils dépassaient les limites assignées, il arrivait à Dom Benoît de sommeiller. Un religieux, bon caricaturiste, avait, pendant une de ces conférences, dessiné le conférencier avec, en dessous, la note: " je serai bref ", et, devant lui, Dom Benoît sommeillant, avec les mots: "il est long». Au fond, la grande horloge indiquait trois bons quarts d'heure d'écoulés. Le même accident lui arrivait encore pendant certains sermons de prédicateurs un peu novices, et aussi pendant la récréation. Avec la vie qu'il menait, on pouvait bien le lui pardonner!

Cette étude de la vie de Dom Benoît nous dépeint assez bien le moine qu'il fut, homme de prière et de mortification dans toute la force du terme. Plus de six heures étaient employées chaque jour à la prière. Homme de travail aussi, qu'il s'agisse de travail intellectuel ou de travail manuel. En vérité, tous ces éléments d'une vie consacrée à Dieu étaient considérés par lui comme nécessaires, et il consacrait aux ceuvres manuelles une heure au moins les jours ordinaires et trois ou quatre heures ou plus les jours de congé et pendant les trois semaines que duraient les vacances scolaires. 
Il aurait été utile de donner d'autres détails sur la vie des C.R.I.C., de dire quelques mots des études et du système d'études, de parler de Dom Benoît écrivain et surtout de sa monumentale Vie de $M^{\text {or }}$ Taché, de nous arrêter quelques instants sur les principaux aspects de son caractère. avec ses qualités et ses défauts, de mentionner aussi certaines erreurs de vision de Dom Gréa et de son plus fidèle disciple, qui ne furent pas toujours assez de leur temps, de rappeler les tristesses qui sensuivirent durant leurs dernières années et de ce que Dom Benoît appelait " la grande épreuve " (nous voulons parler de la transformation de sa communauté en une communauté plus moderne et actuelle), de nous arrêter un instant aux œuvres de la congrégation, qui a conservé ou retrouvé l'esprit de son fondateur... Mais comment dire tant de choses dans une causerie qui se mesure en minutes?

Ce que nous avons dit suffira. croyons-nous. à faire connaître une communauté qui, malgré sa faiblesse et le petit nombre de ses membres. a laissé des traces bienfaisantes dans le diocèse de Saint-Boniface et dans plusieurs autres au Canada. C'est du moins le témoignage que nous rendent aujourd'hui bien des prêtres respectables qui nous ont connus.

J'aurais aimé vous dire ici quelques mots des fondations des C.R.I.C. au Manitoba et dans l'Ouest, de même que dans l'Est, Dom Benoît ayant été nommé vicaire général de Dom Gréa, dès son départ de France, pour toute l'Amérique.

Dès 1891, quelques mois après la fondation de Notre-Dame-deLourdes, la communauté, appelée par $\mathrm{M}^{\mathrm{gr}}$ Duhamel, archevêque d'Ottawa, se voyait confier la paroisse de Nominingue, dans les Laurentides, y succédant aux RR. PP. Jésuites qui l'avaient fondée en 1883. L'Annonciation les eut bientôt comme pasteurs. ainsi que plusieurs autres paroisses ou missions de la même région.

En 1892, les C.R.I.C. allaient aider à Saint-Léon l'abbé Bitsche malade, y faisant le ministère pendant deux ans, et, en 1897, ils y succédaient au curé d'alors, l'abbé Nazaire Pelletier.

Saint-Claude et Saint-Lupicin naissent en 1895 et 1921, ayant été d'abord desservis comme missions par les Pères de Notre-Dame-deLourdes. Saint-Alphonse eut son tour en 1899, puis Mariapolis en 1903. de même que quelques missions dans le voisinage de NotreDame-de-Lourdes ou de Saint-Claude, parmi lesquelles Saint-Daniel, Treherne et Rathwell.

Enfin, quelques autres paroisses leur furent confiées peu après 1900. dans les provinces de Saskatchewan et d'Alberta.

De nos jours, la communauté, dont le développement a été ralenti par les transformations mentionnées précédemment, puis par les 
épreuves de deux guerres mondiales, puis par la crise actuelle des vocations, et qui est réduite à moins de cent membres, n'en continue pas moins ses activités dans cinq pays, à savoir la France, l'Italie, le Canada, le Pérou et l'Angleterre, toujours dans l'unanimité et selon les intentions de l'Église, et ce n'est pas sans un certain plaisir que nous entendons souvent des prêtres nous dire que, dans les secousses actuelles de celle-ci, nous avons su garder l'équilibre et ne pencher ni trop à droite ni trop à gauche.

Nous laisserons au Saint-Père le mot de la fin. Dans une audience accordée le 2 juillet dernier dans la basilique de Saint-Pierre à un groupe de pèlerins français, le pape Paul VI, trop occupé pour donner des audiences privées entre ses voyages à Genève et en Afrique, déclarait:

"Nous sommes heureux de saluer parmi vous les Chanoines réguliers de l'Immaculée Conception, dont le chapitre général (actuellement en cours) a pour but de donner son plein essor à la vie de leurs communautés, centrées principalement sur le culte et l'apostolat paroissial. A ces chers fils, dont l'origine française est maintenant plus que centenaire, Nous exprimons de tout cœur nos encouragements et nos vœux paternels pour un fructueux aggiornamento.»

L'aimable invitation qui m'a été faite de vous entretenir aujourd'hui sur l'œuvre des Chanoines réguliers de l'Immaculée Conception et son aspect particulier ne fait que confirmer ces témoignages concernant le passé et le présent et ce précieux encouragement pour l'avenir, et j'en remercie de tout cœur les organisateurs du présent congrès.

Antoine Champagne, c.r.i.c., Saint-Boniface, Manitoba. 


\section{NOTE}

On a dit parfois que l'œuvre de Dom Benoît colonisateur n'avait pas été un succès. Nous n'admettons pas ce point de vue.

Sans doute avait-il fait de grands rêves, comme toutes les âmes généreuses. Mais pour que l'on puisse parler de faillite, même partielle, il faut que quelque chose ait été entrepris. Or, quand il fut à l'œuvre et se rendit compte de l'immensité du travail à faire, il se contenta de trouver des colons pour le territoire confié à ses soins, c'est-à-dire sa paroisse de Notre-Dame-de-Lourdes, d'où sortirent par démembrement celles de Saint-Claude et de Saint-Lupicin. Dom Benoît ne fut jamais missionnaire-colonisateur dans le sens ordinaire du mot et n'a pas entrepris de l'être. Il n'y a donc pas lieu de le comparer à d'autres prêtres qui ont amené dans l'Ouest canadien plus de colons que lui, quelquefois avec un résultat plutôt douteux.

De plus, dès 1895, ayant amené ou fait organiser cinq convois de colons français et suisses, il jugea que cela était suffisant et que ceux déjà arrivés, dont plusieurs commençaient à prospérer, attireraient leurs parents et amis et achèveraient l'œuvre commencée. Une excellente sélection se fit ainsi, qui élimina les indésirables. Cette politique sage et réaliste a porté ses fruits et on peut en constater aujourd'hui comme alors les bons résultats: les paroisses de Notre-Dame-de-Lourdes et de Saint-Claude sont aujourd'hui de belles et florissantes paroisses.

\section{RÉFÉRENCES BIBLIOGRAPHIQUES}

1. Vernet, M ${ }^{g r}$ Félix, Dom Gréa, 1828-1917, Paris, Labergerie, 1937.

2. Ouvrages de Dom GrÉA:

- L'Eglise et sa divine constitution, La Bonne Presse, 1885, 1907, et Casterman, 1965 ;

- La sainte liturgie, La Bonne Presse, Paris, 1907; et autres écrits.

3. Ouvrages de Dom Benoît:

- Vie de Mor Taché, 2 vol., Montréal, Beauchemin, 1904;

- Nombreux articles dans des journaux et revues, sur les questions scolaires, les C.R.I.C., Notre-Dame-de-Lourdes, etc., en particulier dans * Le Manitoba *, Les Cloches de Saint-Boniface;

- Vie de Dom Gréa, manuscrit.

4. Livres paroissiaux de Notre-Dame-de-Lourdes:

- Baptêmes, mariages, sépultures;

- Liber animarum;

- Codex historicus.

5. Archives de l'archevêché de Saint-Boniface: lettres et divers.

6. Archives des C.R.I.C. à Rome.

7. Bulletin C.R.I.C.

8. Champagne, Antoine, Les commencements de Notre-Dame-de-Lourdes, C.R.I.C., 1943, manuscrit.

9. L'auteur de la précédente étude ou causerie ayant vécu plus de huit ans dans l'intimité de Dom Benoît et lui ayant servi longtemps de secrétaire particulier, puis s'étant trouvé pendant douze ans sous les nouvelles règles des C.R.I.C., à Rome et en France, peut témoigner - mieux que plusieurs autres - de l'objectivité des faits qui y sont relatés. 